\title{
Attitude Stabilization of a Rigid Spacecraft Using Two Momentum Wheel Actuators
}

\author{
Hariharan Krishnan* \\ National University of Singapore, 0511 Singapore \\ N. Harris McClamroch ${ }^{\dagger}$ \\ University of Michigan, Ann Arbor, Michigan 48109-2140 \\ and \\ Mahmut Reyhanoglu ${ }^{\ddagger}$ \\ King Fahd University of Petroleum and Minerals, Dhahran 31261, Saudi Arabia
}

\begin{abstract}
It is well known that three momentum wheel actuators can be used to control the attitude of a rigid spacecraft and that arbitrary reorientation maneuvers of the spacecraft can be accomplished using smooth feedback. If failure of one of the momentum wheel actuators occurs, we demonstrate that two momentum wheel actuators can be used to control the attitude of a rigid spacecraft and that arbitrary reorientation maneuvers of the spacecraft can be accomplished. Although the complete spacecraft equations are not controllable, the spacecraft equations are controllable under the restriction that the total angular momentum vector of the system is zero. The spacecraft dynamics under such a restriction cannot be asymptotically stabilized to any equilibrium attitude using a timeinvariant continuous feedback control law, but discontinuous feedback control strategies are constructed that stabilize any equilibrium attitude of the spacecraft in finite time. Consequently, reorientation of the spacecraft can be accomplished using discontinuous feedback control.
\end{abstract}

\section{Introduction}

$\mathbf{W}$ E consider the attitude control of a spacecraft modeled as a rigid body. It is well known that three actuators, either gas jets or momentum wheels, can be used to control the attitude of a rigid spacecraft and that arbitrary reorientation maneuvers of the spacecraft can be accomplished using smooth feedback. ${ }^{1-7}$ If failure of one of the actuators occurs, then one is left with only two actuators. In this paper, the attitude stabilization problem of a rigid spacecraft using only two control torques supplied by momentum wheel actuators is considered. Since we are considering a spacebased system, the problem considered here, namely, the attitude stabilization of a spacecraft operating in an actuator failure mode, is an important control problem. It is assumed that the center of mass of the system consisting of the spacecraft and the momentum wheel actuators is fixed in space.

Attitude stabilization of a rigid spacecraft using two momentum wheel actuators is not a mature subject in the literature. Controllability results for a rigid spacecraft controlled by momentum wheel actuators are presented in Ref. 8 . We mention that most of the previous researchers have considered the problem of controlling a rigid spacecraft using less than three gas jet actuators. ${ }^{8-23}$ Attitude stabilization of a rigid spacecraft using two gas jet actuators is considered in Refs. 8-13. References 14-23 consider the stabilization of the angular velocity equations of a rigid spacecraft using less than three gas jet actuators. In Ref. 19, an expression is given for the geometric phase ${ }^{24}$ of the attitude of a rigid body with two momentum wheel actuators under the assumption that the total angular momentum vector of the system is zero. It is mentioned that the geometric phase can be used to compensate for the attitude drift of the rigid body. However, explicit control methods are not presented. Related work on spacecraft control appears in Refs. 25 and 26, but they do not consider the same problem being studied in this paper.

We consider the attitude stabilization of a spacecraft using control torques supplied by two momentum wheel actuators about axes

Received Feb 16, 1993; revision received May 11, 1994; accepted for publication Sept. 6, 1994. Copyright (C) 1995 by the American Institute of Aeronautics and Astronautics, Inc. All rights reserved.

* Lecturer, Department of Mechanical and Production Engineering.

Trofessor, Department of Aerospace Engineering. Member AIAA.

*Assistant Professur, Department of Mechanical Engineering. spanning a two-dimensional plane orthogonal to a principal axis of the spacecraft. The first-order linearization of the complete spacecraft dynamic equations at any equilibrium attitude has an uncontrollable eigenvalue at the origin. Consequently, controllability and stabilizability properties of the spacecraft cannot be inferred using classical linearization ideas. The complete spacecraft dynamics is, in fact, not controllable. Under the assumption that the total angular momentum vector of the system is zero, the spacecraft dynamics is locally controllable at any equilibrium attitude. The spacecraft dynamics under such a restriction cannot be asymptotically stabilized to any equilibrium attitude using time-invariant continuous feedback. Nevertheless, two different discontinuous feedback control strategies are constructed that achieve reorientation of the spacecraft in finite time. Using the concept of geometric phase, ${ }^{24}$ a discontinuous feedback control strategy is presented based on the nonholonomic control theory in Ref. 27. An alternate discontinuous feedback control strategy, based on the fact that rigid-body rotations do not commute, is also presented.

This paper is based on our earlier work presented in Ref. 10 and is a companion to Refs. 11 and 12 , which treat the attitude stabilization of a rigid spacecraft using two gas jet actuators.

\section{Kinematic and Dynamic Equations}

The orientation of a rigid spacecraft can be specified using various parametrizations of the special orthogonal group $S O(3)$. Here we use the 3-2-1 Euler angle convention for parametrizing the orientation of the rigid spacecraft. ${ }^{28}$ Consider an inertial $X_{1} X_{2} X_{3}$ coordinate frame; let $x_{1} x_{2} x_{3}$ be a coordinate frame aligned with the principal axes of the spacecraft with origin at the center of mass of the spacecraft. If the two frames are initially coincident, a series of three rotations about the body axes performed in the proper sequence is sufficient to allow the spacecraft to reach any orientation. The three rotations are a positive rotation of frame $X_{1} X_{2} X_{3}$ by angle $\psi$ about the $X_{3}$ axis (let $x_{1}^{\prime} x_{2}^{\prime} x_{3}^{\prime}$ denote the resulting coordinate frame), a positive rotation of frame $x_{1}^{\prime} x_{2}^{\prime} x_{3}^{\prime}$ by angle $\theta$ about the $x_{2}^{\prime}$ axis (let $x_{1}^{\prime \prime} x_{2}^{\prime \prime} x_{3}^{\prime \prime}$ denote the resulting frame), and a positive rotation of frame $x_{1}^{\prime \prime} x_{2}^{\prime \prime} x_{3}^{\prime \prime}$ by angle $\phi$ about the $x_{1}^{\prime \prime}$ axis (let $x_{1} x_{2} x_{3}$ denote the final coordinate frame). The corresponding rotation matrix is denoted as $R(\psi, \theta, \phi)$, where $\psi, \theta$, and $\phi$ are the Euler angles. We assume that the Euler angles are limited to the ranges $-\pi \leq \psi<\pi,-\pi / 2<\theta<\pi / 2,-\pi \leq \phi \leq \pi$. The 3-2-1 


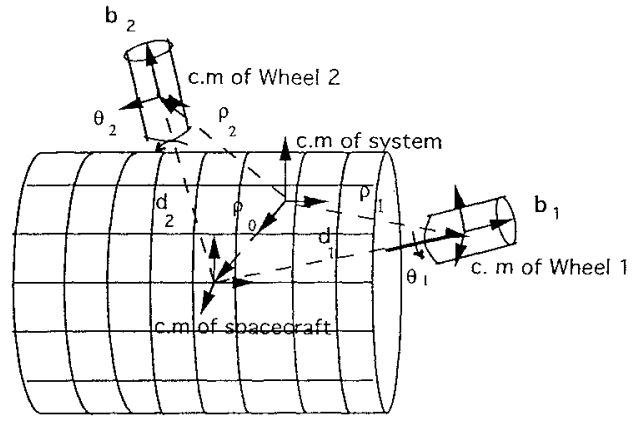

Fig. 1 Spacecraft system.

Euler angle system is commonly used to represent the orientation of aerospace systems. ${ }^{28}$ Moreover, the 3-2-1 Euler angle system has a singularity when $\theta= \pm \pi / 2$, whereas a number of other Euler angle systems have a singularity when $\theta=0$. Since it is conventional in control theory to formulate the stabilization problem about the origin, many of the Euler angle systems with singularity at $\theta=0$ would render the formulation ill posed. The Euler angle system used here has no additional restrictions in comparison to other Euler angle systems, and the control strategy developed here can be modified to suit other Euler angle representations as well.

Suppose $\omega_{1}, \omega_{2}, \omega_{3}$ are the principal axis components of the absolute angular velocity vector $\omega$ of the spacecraft. Then we have ${ }^{28}$

$$
\begin{gathered}
\dot{\phi}=\omega_{1}+\omega_{2} \sin \phi \tan \theta+\omega_{3} \cos \phi \tan \theta \\
\dot{\theta}=\omega_{2} \cos \phi-\omega_{3} \sin \phi \\
\dot{\psi}=\omega_{2} \sin \phi \sec \theta+\omega_{3} \cos \phi \sec \theta
\end{gathered}
$$

Next we consider the dynamic equations that describe the evolution of the angular velocity components of the spacecraft. Consider two momentum wheel actuators, wheels 1 and 2 , spinning about axes defined by unit vectors $\boldsymbol{b}_{1}, \boldsymbol{b}_{2}$ fixed in the spacecraft (see Fig. 1). The center of mass of wheel 1 lies on the axis defined by $b_{1}$, and the center of mass of wheel 2 lies on the axis defined by $\boldsymbol{b}_{2}$. Control torques $-\bar{u}_{1}$ and $-\bar{u}_{2}$ are supplied to wheels 1 and 2 about the axis defined by $\boldsymbol{b}_{1}$ and $\boldsymbol{b}_{2}$, respectively, by motors fixed in the spacecraft. Consequently, equal and opposite torques $\bar{u}_{1}$ and $\bar{u}_{2}$ are exerted by wheels 1 and 2, respectively, on the spacecraft. We assume that $\boldsymbol{b}_{1}$ defines a principal axis for wheel 1 , which is symmetric about that axis, and $\boldsymbol{b}_{2}$ defines a principal axis for wheel 2 , which is symmetric about that axis. Further $\boldsymbol{b}_{1}$ and $\boldsymbol{b}_{2}$ span a two-dimensional plane that is orthogonal to a principal axis of the spacecraft. Without loss of generality, $b_{1}$ and $b_{2}$ are assumed to be of the form

$$
\begin{aligned}
& \boldsymbol{b}_{1}=\left(b_{1 x}, b_{1 y}, 0\right)^{T} \\
& \boldsymbol{b}_{2}=\left(b_{2 x}, b_{2 y}, 0\right)^{T}
\end{aligned}
$$

The mass of the spacecraft, wheel 1 , and wheel 2 are denoted as $m_{0}, m_{1}$, and $m_{2}$, respectively, and $\boldsymbol{\rho}_{0}, \boldsymbol{\rho}_{1}, \boldsymbol{\rho}_{2}$ denote the position vectors of the center of mass of the spacecraft, wheel 1 , and wheel 2 , respectively, with respect to the center of mass of the whole system. Thus from the location of the wheels

$$
\begin{aligned}
& \rho_{1}=\rho_{0}+d_{1} b_{1} \\
& \rho_{2}=\rho_{0}+d_{2} b_{2}
\end{aligned}
$$

where $d_{1}, d_{2}$ are constants that are the distances of wheels 1 and 2 from the center of mass of the spacecraft along the unit vectors $b_{1}$ and $\boldsymbol{b}_{2}$, respectively. Since, by the definition of center of mass,

$$
\sum_{i=0}^{2} m_{i} \rho_{i}=0
$$

further manipulation of Eqs. (6-8) gives expressions for $\rho_{0}, \rho_{1}$, and $\rho_{2}$, which we denote as $\rho_{i}=\left(\rho_{i x}, \rho_{i,}, 0\right)^{T}, i=0,1,2$. The total angular momentum vector of the system is given, in the spacecraft body frame, by

$$
R(\psi, \theta, \phi) H=J \omega+v
$$

where

$$
\begin{gathered}
\boldsymbol{J}=\left[\boldsymbol{I}_{0}+\sum_{i=0}^{2} \tilde{\boldsymbol{I}}_{i}+\sum_{i=1}^{2}\left(\boldsymbol{I}_{i}-\underline{\boldsymbol{I}}_{i}\right)\right] \\
\overrightarrow{\boldsymbol{I}}_{i}=m_{i}\left[\begin{array}{ccc}
\rho_{i y}^{2} & -\rho_{i x} \rho_{i y} & 0 \\
-\rho_{i x} \rho_{i y} & \rho_{i x}^{2} & 0 \\
0 & 0 & \rho_{i x}^{2}+\rho_{i y}^{2}
\end{array}\right], \quad i=0,1,2 \\
\underline{\boldsymbol{I}}_{1}=\boldsymbol{b}_{1} \boldsymbol{b}_{1}^{T} j_{1} \\
\overrightarrow{\boldsymbol{I}}_{2}=\boldsymbol{b}_{2} \boldsymbol{b}_{2}^{T} \dot{j}_{2} \\
v=\underline{\boldsymbol{I}}_{1}\left(\boldsymbol{\omega}+\boldsymbol{b}_{1} \dot{\boldsymbol{\theta}}_{\mathbf{1}}\right)+\underline{\boldsymbol{I}}_{2}\left(\boldsymbol{\omega}+\boldsymbol{b}_{2} \dot{\boldsymbol{\theta}}_{2}\right)
\end{gathered}
$$

where $\boldsymbol{I}_{0}, \boldsymbol{I}_{1}$, and $\boldsymbol{I}_{2}$ denote the inertial tensors of the spacecraft, wheel 1 , and wheel 2 , respectively, $j_{1}$ is the moment of inertia of wheel 1 about the axis defined by $\boldsymbol{b}_{1}, j_{2}$ is the moment of inertia of wheel 2 about the axis defined by $\boldsymbol{b}_{2}$, and $\theta_{1}, \theta_{2}$ are the angles of rotation of wheels 1 and 2 about the axes defined by $\boldsymbol{b}_{1}$ and $\boldsymbol{b}_{2}$, respectively. The vector $v$ in Eq. (9), which is defined by Eq. (14), is the sum of the angular momentum of wheels 1 and 2 relative to the spacecraft. Here $\boldsymbol{H}$ denotes the angular momentum vector of the system expressed in the inertial coordinate frame. The angular momentum vector $\boldsymbol{H}$ is a constant since there is no external moment about the center of mass of the system. Suppose $\bar{u}_{1}$ and $\bar{u}_{2}$ are the control torques; then

$$
\dot{v}=-\left(b_{1} \bar{u}_{1}+b_{2} \bar{u}_{2}\right)
$$

Differentiating Eq. (9) with respect to time, we obtain

$$
J \dot{\omega}=S(\omega) \boldsymbol{R}(\psi, \boldsymbol{\theta}, \phi) \boldsymbol{H}+\boldsymbol{b}_{1} \bar{u}_{1}+\boldsymbol{b}_{2} \bar{u}_{2}
$$

where

$$
\boldsymbol{S}(\boldsymbol{\omega})=\left[\begin{array}{ccc}
0 & \omega_{3} & -\omega_{2} \\
-\omega_{3} & 0 & \omega_{1} \\
\omega_{2} & -\omega_{1} & 0
\end{array}\right]
$$

Note that

$$
\begin{gathered}
\boldsymbol{I}_{0}=\operatorname{diag}\left(I_{01}, I_{02}, I_{03}\right) \\
\boldsymbol{I}_{1}=\operatorname{block} \operatorname{diag}\left(I_{11}, I_{12}\right) \\
\boldsymbol{I}_{2}=\operatorname{block} \operatorname{diag}\left(\boldsymbol{I}_{21}, I_{22}\right)
\end{gathered}
$$

where $I_{11}, I_{21}$ are invertible $2 \times 2$ matrices, $I_{01}, I_{02}, I_{03}, I_{12}, I_{22}$ are nonzero real numbers, and therefore $J$ is a positive-definite matrix of the form

$$
\boldsymbol{J}=\text { block diag }\left(\boldsymbol{J}_{1}, \boldsymbol{J}_{2}\right)
$$

where $J_{1}$ is an invertible $2 \times 2$ matrix and $J_{2}$ is a nonzero real number.

\section{Controllability and Stabilization Properties}

In this section we consider the controllability and stabilizability properties of the spacecraft dynamics controlled by two momentum wheel actuators. Define

$$
\left[\begin{array}{l}
u_{1} \\
u_{2}
\end{array}\right]=J_{1}^{-1}\left[\begin{array}{ll}
b_{1 x} & b_{2 x} \\
b_{1 y} & b_{2 y}
\end{array}\right]\left[\begin{array}{l}
\bar{u}_{1} \\
\bar{u}_{2}
\end{array}\right]
$$


From Sec. II the complete spacecraft dynamics can be rewritten as

$$
\begin{gathered}
\dot{\boldsymbol{\omega}}=\left[\begin{array}{cc}
J_{1}^{-1} & \mathbf{0}_{(2 \times 1)} \\
\mathbf{0}_{(1 \times 2)} & J_{2}^{-1}
\end{array}\right] \boldsymbol{S}(\boldsymbol{\omega}) \boldsymbol{R}(\psi, \boldsymbol{\theta}, \phi) \boldsymbol{H}+\left[\begin{array}{c}
u_{1} \\
u_{2} \\
0
\end{array}\right] \\
\dot{\phi}=\omega_{1}+\omega_{2} \sin \phi \tan \theta+\omega_{3} \cos \phi \tan \theta \\
\dot{\theta}=\omega_{2} \cos \phi-\omega_{3} \sin \phi \\
\dot{\psi}=\omega_{2} \sin \phi \sec \theta+\omega_{3} \cos \phi \sec \theta
\end{gathered}
$$

where $\boldsymbol{H}$ is a constant vector.

The first-order linearization of the complete spacecraft dynamic Eqs. (19-22) at any equilibrium attitude has an uncontrollable eigenvalue at the origin. Consequently, the controllability and stabilizability properties of the complete spacecraft dynamics cannot be inferred using classical linearization ideas. However, from Eqs. (4), (5), and (12-14) and the definition

$$
c=(0,0,1)^{T}
$$

we have $c^{T} v=0$. Therefore from Eq. (9) we have

$$
c^{T} \boldsymbol{R}(\psi, \theta, \phi) H=c^{T} J \omega
$$

Since $\boldsymbol{H}$ is a constant vector, this equation represents a constraint on the motion of the spacecraft irrespective of the controls applied. Thus the complete spacecraft dynamics is not completely controllable. Therefore we ask the following question: What restricted control and stabilization properties of the spacecraft can be demonstrated in this case? Our analysis begins by demonstrating that, under an appropriate restriction of interest, the spacecraft equations have restricted controllability and stabilizability properties.

Consider Eqs. (19-22) and suppose the angular momentum vector $\boldsymbol{H}$ of the system is zero. From Eqs. (17), (23), and (24) it follows that the angular velocity component of the spacecraft about the uncontrolled princip:!' axis is identically zero, i.e., $\omega_{3} \equiv 0$. Under such a restriction, the reduced spacecraft dynamics are described by

$$
\begin{gathered}
\dot{\omega}_{1}=u_{1} \\
\dot{\omega}_{2}=u_{2} \\
\dot{\phi}=\omega_{1}+\omega_{2} \sin \phi \tan \theta \\
\dot{\theta}=\omega_{2} \cos \phi \\
\dot{\psi}=\omega_{2} \sin \phi \sec \theta
\end{gathered}
$$

Notice that the first-order linearization of Eqs. (25-29) at any equilibrium has an uncontrollable eigenvalue at the origin. Therefore analysis of the controllability and stabilizability properties of the reduced spacecraft dynamics requires inherently nonlinear techniques. Equations (25-29) are of the form

$$
\dot{\boldsymbol{x}}=f(x)+g_{1} u_{1}+g_{2} u_{2}
$$

where $\boldsymbol{x}=\left(\omega_{1}, \omega_{2}, \phi, \theta, \psi\right)^{T} \in \boldsymbol{M}$ where $\boldsymbol{M}$ denotes the set

$$
\begin{gathered}
\boldsymbol{M}=\left\{x: \omega_{i} \in R, i=1,2, \phi, \psi \in[-\pi, \pi],\right. \\
\theta \in(-0.5 \pi, 0.5 \pi)\}
\end{gathered}
$$

and $\boldsymbol{f}(\boldsymbol{x}), \boldsymbol{g}_{1}, \boldsymbol{g}_{2}$ are vector fields defined appropriately on $\boldsymbol{M}$.

Let $\boldsymbol{r}(p, t)$ denote the set of reachable states from the initial state $p$ in time exactly $t$ for Eq. (30). The following definition is standard (see Refs. 29 and 30).

Definition 1. Consider the system (30) and let $\boldsymbol{p} \in \boldsymbol{M}$ :

1) The system is said to be accessible at $p$ if, for any $T>$ $0, \cup_{t \leq r} \boldsymbol{r}(\boldsymbol{p}, t)$ has a nonvoid interior with respect to $\boldsymbol{M}$. If this holds for all $p \in M$, then the system is said to be accessible.

2) The system is said to be small time locally controllable (STLC) at $\boldsymbol{p}$ if, for any $T>0, p$ is an interior point of $\cup_{t \leq T} r(p, t)$.
Define the Lie bracket of two vector fields $f_{1}(\boldsymbol{x})$ and $\boldsymbol{f}_{2}(\boldsymbol{x})$ as $^{29}$

$$
\left[f_{1}, f_{2}\right]=\frac{\partial f_{2}(x)}{\partial x} f_{1}(x)-\frac{\partial f_{1}(x)}{\partial x} f_{2}(x)
$$

The following results follow directly based on an analysis similar to that in Ref. 27.

Theorem 1. The reduced dynamics of a spacecraft controlled by two momentum wheel actuators are STLC at any equilibrium.

Proof. The vector fields $\boldsymbol{g}_{1}, \boldsymbol{g}_{2},\left[\boldsymbol{g}_{1}, \boldsymbol{f}\right],\left[\boldsymbol{g}_{2}, \boldsymbol{f}\right]$, $\left[\left[\boldsymbol{g}_{2}, \boldsymbol{f}\right],\left[\boldsymbol{g}_{1}, \boldsymbol{f}\right]\right]$ span a five dimensional space at every $\boldsymbol{x} \in \boldsymbol{M}$. Thus the accessibility Lie algebraic rank condition ${ }^{29}$ is satisfied and hence the reduced spacecraft dynamics are accessible, which is a necessary condition for STLC. Following Ref. 30 let $\boldsymbol{B r}(\boldsymbol{x})$ denote the smallest Lie algebra of vector fields containing $\boldsymbol{f}, \boldsymbol{g}_{1}, \boldsymbol{g}_{2}$. Let $\boldsymbol{B}$ be any bracket in $\boldsymbol{B r}(\boldsymbol{x})$. Now denote $\delta^{0}(\boldsymbol{B}), \delta^{\mathrm{l}}(\boldsymbol{B}), \delta^{2}(\boldsymbol{B})$ as the number of occurrences of the vector fields $f, g_{1}, g_{2}$, respectively, in the bracket $\boldsymbol{B}$. The degree of $\boldsymbol{B}$ is equal to the value of $\sum_{i=0}^{2} \delta^{i}(\boldsymbol{B})$. The Sussman condition for small time local controllability at any equilibrium is that the so-called bad brackets, the brackets with $\delta^{0}$ odd and $\delta^{1}, \delta^{2}$ even, must be a linear combination of brackets of lower degree at that equilibrium. From the proof of accessibilty given above it is clear that any bracket of degree greater than 4 can be expressed as a linear combination of lower order brackets at any equilibrium. Moreover, the degree of a bad bracket must necessarily be odd. The bad bracket of degree 1 is $f$, which vanishes at any equilibrium. The bad brackets of degree 3 are $\left[g_{1},\left[g_{1}, f\right]\right]$ and $\left[g_{2},\left[g_{2}, f\right]\right]$ and both are identically zero vector fields. Thus the sufficient condition in Ref. 30 is satisfied at any equilibrium, and therefore the reduced spacecraft dynamics are STLC.

Theorem 2. The reduced dynamics of a spacecraft controlled by two momentum wheel actuators cannot be asymptotically stabilized to any equilibrium using a time-invariant continuous feedback control law, but the reduced dynamics can be asymptotically stabilized to any equilibrium using a piecewise continuous feedback control law.

Proof. Brockett's neccesary condition for the existence of a time-invariant continuous feedback control law that asymptotically stabilizes any equilibrium of Eq. (30) is that the image of the map

$$
\left(x, u_{1}, u_{2}\right) \rightarrow f(x)+g_{1} u_{1}+g_{2} u_{2}
$$

must contain a neighborhood of the origin. ${ }^{17,31,32}$ Note that the system defined by Eqs. (25-29) is a dynamic extension ${ }^{31.32}$ of the system defined by Eqs. (27-29) with $\omega_{1}, \omega_{2}$ as its control inputs, which can be expressed in the form

$$
\dot{\boldsymbol{y}}=\boldsymbol{f}_{1}(\boldsymbol{y}) \omega_{1}+\boldsymbol{f}_{2}(\boldsymbol{y}) \omega_{2}
$$

where $\boldsymbol{y}=(\phi, \theta, \psi)^{T}$ and $f_{1}, f_{2}$ are defined appropriately. At any equilibrium $\boldsymbol{y}_{e}=\left(\phi_{e}, \theta_{e}, \psi_{e}\right)^{T}$,

$$
\operatorname{rank}\left\{f_{1}\left(y_{e}\right), f_{2}\left(y_{e}\right)\right\}=2
$$

The number of inputs in Eq. (34) is 2, whereas the dimension of the state space is 3. Therefore from Refs. 17 and 34 the system does not satisfy Brockett's necessary condition at any equilibrium. From Refs. 31 and 32, it is clear that the topological obstruction to stabilizability discovered by Brockett is clearly preserved under dynamic extension, i.e., if Eqs. (27-29) do not satisfy Brockett's necessary condition at $\left(\phi_{e}, \theta_{e}, \psi_{e}\right)^{T}$, then Eqs. (25-29) do not satisfy Brockett's necessary condition at $\left(0,0, \phi_{e}, \theta_{e}, \psi_{e}\right)^{T}$. Thus the reduced spacecraft dynamics cannot be asymptotically stabilized to any equilibrium using a time-invariant continuous feedback control law. The second part of the theorem is a consequence of a result due to small time local controllability in Ref. 33, which states that if a system is real analytic and STLC at any equilibrium, then there exists a piecewise continuous feedback controller that asymptotically stabilizes the system to that equilibrium.

Note that in Theorem 2, by time-invariant feedback control law we mean any feedback control law that does not depend explicitly on time. Since Eqs. (25-29) are STLC at any equilibrium, from Definition 1 it is clear that starting from any equilibrium an open neighborhood of the equilibrium can be reached by trajectories of 
the system for any time $T>0$. Since the system is real analytic, the argument can be reversed (see Refs. 30, 31, and 33). Thus for any $T>0$ there exists an open neighborhood of any equilibrium starting from which the equilibrium can be reached within time $T$. Moreover, Theorem 2 states that the equilibrium can be asymptotically stabilized using piecewise continuous feedback. This is a local property. However, notice that Eqs. (25-29) have a large set of equilibrium points that are all connected. Typically any state of the form $\omega_{1}=0, \omega_{2}=0, \phi, \theta, \psi$ are arbitrary is an equilibrium. Thus if one knows how to steer any given state in $\boldsymbol{M}$ to some equilibrium, and if one knows how to steer between equilibrium points, then the asymptotic stabilization can be made global by patching together the various piecewise continuous feedback control laws. In the next section two different feedback control strategies are developed that asymptotically stabilize the reduced spacecraft dynamics from any initial state in $\boldsymbol{M}$ to any equilibrium attitude in $\boldsymbol{M}$.

We conclude this section by summarizing the implications of the properties stated above. Suppose the angular momentum vector $\boldsymbol{H}$ is zero. Then the spacecraft controlled by two momentum wheel actuators can be controlled to any equilibrium attitude, but the feedback control law must necessarily be discontinuous. Thus reorientation of the spacecraft can be achieved under the restriction $\boldsymbol{H}=0$; If $\boldsymbol{H} \neq \mathbf{0}$, reorientation of the spacecraft to an equilibrium attitude cannot be achieved.

\section{Feedback Stabilization Algorithms}

We restrict our study to the class of discontinuous feedback controllers in order to asymptotically stabilize the reduced spacecraft dynamics described by state Eqs. (25-29). An algorithm generating a discontinuous feedback control that asymptotically stabilizes any equilibrium can be constructed, as suggested by the controllability properties of the system. Without loss of generality, we assume that the equilibrium to be stabilized is the origin. We present two different discontinuous control strategies that stabilize the origin of Eqs. (25-29) in finite time.

\section{A. Feedback Stabilization Based on Nonholonomic Control Theory} Consider a diffeomorphism defined by

$$
\begin{gathered}
y_{1}=\cos \phi \ln (\sec \theta+\tan \theta)+\psi \sin \phi \\
y_{2}=\omega_{2} \sec \theta-y_{4} y_{5} \\
y_{3}=\phi \\
y_{4}=\omega_{1}+\omega_{2} \sin \phi \tan \theta \\
y_{5}=\sin \phi \ln (\sec \theta+\tan \theta)-\psi \cos \phi
\end{gathered}
$$

If we now define the feedback relations

$$
\begin{aligned}
& {\left[\begin{array}{l}
u_{1} \\
u_{2}
\end{array}\right]=\left[\begin{array}{cc}
-\sin \phi \sin \theta & \left(1-y_{5} \sin \phi \sin \theta\right) \\
\cos \theta & y_{5} \cos \theta
\end{array}\right]} \\
& \quad \times\left\{\left(\begin{array}{l}
v_{1} \\
v_{2}
\end{array}\right)+\left(\begin{array}{c}
\cos \phi y_{5} \sin \phi \sec ^{2} \theta \omega_{2}^{2} \\
0
\end{array}\right)\right. \\
& \left.-\left(\begin{array}{c}
-y_{4}^{2} y_{1}+\cos \phi\left(\sec \theta \tan \theta \omega_{2}^{2}-y_{5} y_{4} \tan \theta \omega_{2}\right) \\
\cos \phi\left(y_{4} \tan \theta \omega_{2}+\sin \phi \sec ^{2} \theta \omega_{2}^{2}\right)
\end{array}\right)\right\}
\end{aligned}
$$

then the reduced spacecraft dynamics (25)-(29) are described in the new variables by the normal form equations

$$
\begin{gathered}
\dot{y}_{1}=y_{2} \\
\dot{y}_{2}=v_{1} \\
\dot{y}_{3}=y_{4} \\
\dot{y}_{4}=v_{2} \\
\dot{y}_{5}=y_{4} y_{1}
\end{gathered}
$$

Notice that Eqs. (35-39) define a global diffeomorphism, and therefore $\omega_{1}=\omega_{2}=\phi=\theta=\psi=0$ implies that $y_{1}=y_{2}=y_{3}=y_{4}=$ $y_{5}=0$ and vice versa (see Appendix). Hence asymptotic stabilization of Eqs. (25-29) to the origin is equivalent to asymptotic stabilization of the normal-form equations $(41-45)$ to the origin; hence we consider asymptotic stabilization of the normal-form equations. The normal-form equations $(41-45)$ are in a familiar form that has been studied in Ref. 27 and therefore can be stabilized by the following discontinuous control strategy:

1) Transfer the initial state of the normal-form equations (41-45) to the equilibrium state $\left(0,0,0,0, y_{5}^{1}\right)$, for some $y_{5}^{1}$, in finite time.

2) Next, traverse a closed path $\gamma$ in the $\left(y_{1}, y_{3}\right)$ space in finite time, where the path $\gamma$ is selected to satisfy

$$
-y_{5}^{1}=\int_{\gamma} y_{1} d y_{3}
$$

This transfers the state $\left(0,0,0,0, y_{5}^{1}\right)$ to the origin in finite time.

Here we consider a rectangular path $\gamma$ in the $\left(y_{1}, y_{3}\right)$ space formed by line segments from $(0,0)$ to $\left(y_{1}^{*}, 0\right)$, from $\left(y_{1}^{*}, 0\right)$ to $\left(y_{1}^{*}, y_{3}^{*}\right)$, from $\left(y_{1}^{*}, y_{3}^{*}\right)$ to $\left(0, y_{3}^{*}\right)$, and from $\left(0, y_{3}^{*}\right)$ to $(0,0)$. For such a path, the line integral in Eq. (46) can be explicitly evaluated as $y_{1}^{*} y_{3}^{*}$ so that Eq. (46) becomes

$$
-y_{5}^{1}=y_{1}^{*} y_{3}^{*}
$$

and the parameters $y_{1}^{*}$ and $y_{3}^{*}$ specifying the particular rectangular path are chosen to satisfy the above equation.

Throughout, assume $k>0$, and define

$$
\begin{aligned}
& G\left(x_{1}, x_{2}\right)= \\
& \left\{\begin{array}{l}
k \text { if }\left\{x_{1}+\frac{x_{2}\left|x_{2}\right|}{2 k}>0\right\} \text { or }\left\{x_{1}+\frac{x_{2}\left|x_{2}\right|}{2 k}=0 \text { and } x_{2}>0\right\} \\
-k \text { if }\left\{x_{1}+\frac{x_{2}\left|x_{2}\right|}{2 k}<0\right\} \text { or }\left\{x_{1}+\frac{x_{2}\left|x_{2}\right|}{2 k}=0 \text { and } x_{2}<0\right\} \\
0 \text { if }
\end{array}\right.
\end{aligned}
$$

The function $G\left(x_{1}, x_{2}\right)$ is the time optimal control law for a double integrator. We use the well-known property (see Ref. 35, pp. 507514) that any initial state of the system

$$
\begin{gathered}
\dot{x}_{1}=x_{2} \\
\dot{x}_{2}=-G\left(x_{1}-\bar{x}_{1}, x_{2}\right)
\end{gathered}
$$

is transferred to the final state $\left(\bar{x}_{1}, 0\right)$ in a finite time.

We now present a specific feedback control algorithm that stabilizes the spacecraft to the origin in finite time; this feedback control algorithm implements the approach just described.

Maneuver 1 . Apply

$$
\begin{aligned}
& v_{1}=-G\left(y_{1}, y_{2}\right) \\
& v_{2}=-G\left(y_{3}, y_{4}\right)
\end{aligned}
$$

until $\left(y_{1}, y_{2}, y_{3}, y_{4}, y_{5}\right)=\left(0,0,0,0, y_{5}^{1}\right)$ where $y_{5}^{1}$ is arbitrary; then go to maneuver 2 .

Maneuver 2 . If $y_{5}^{1} \geq 0$, choose $y_{1}^{*}=-y_{3}^{*}=\sqrt{y_{5}^{1}}$; else choose $y_{1}^{*}=y_{3}^{*}=\sqrt{-y_{5}^{1}}$. Apply

$$
\begin{gathered}
v_{1}=-G\left(y_{1}-y_{1}^{*}, y_{2}\right) \\
v_{2}=-G\left(y_{3}, y_{4}\right)
\end{gathered}
$$

until $\left(y_{1}, y_{2}, y_{3}, y_{4}, y_{5}\right)=\left(y_{1}^{*}, 0,0,0, y_{5}^{1}\right) ;$ then go to maneuver 3 . Maneuver 3. Apply

$$
\begin{aligned}
& v_{1}=-G\left(y_{1}-y_{1}^{*}, y_{2}\right) \\
& v_{2}=-G\left(y_{3}-y_{3}^{*}, y_{4}\right)
\end{aligned}
$$

until $\left(y_{1}, y_{2}, y_{3}, y_{4}, y_{5}\right)=\left(y_{1}^{*}, 0, y_{3}^{*}, 0,0\right)$; then go to maneuver 4 . 
Maneuver 4. Apply

$$
\begin{gathered}
v_{1}=-G\left(y_{1}, y_{2}\right) \\
v_{2}=-G\left(y_{3}-y_{3}^{*}, y_{4}\right)
\end{gathered}
$$

until $\left(y_{1}, y_{2}, y_{3}, y_{4}, y_{5}\right)=\left(0,0, y_{3}^{*}, 0,0\right)$; then go to maneuver 5 . Maneuver 5. Apply

$$
\begin{aligned}
& v_{1}=-G\left(y_{1}, y_{2}\right) \\
& v_{2}=-G\left(y_{3}, y_{4}\right)
\end{aligned}
$$

until $\left(y_{1}, y_{2}, y_{3}, y_{4}, y_{5}\right)=(0,0,0,0,0)$; then go to maneuver 2 .

It can be verified that the execution of maneuver 1 transfers the initial state of the normal-form equations to the equilibrium state $\left(0,0,0,0, y_{5}^{1}\right)$ for some $y_{5}^{1}$ in finite time. Subsequent execution of maneuvers $2-5$ then transfers the state $\left(0,0,0,0, y_{5}^{1}\right)$ to the origin in finite time. This control algorithm is nonclassical and involves switching between various feedback functions. Justification that it stabilizes the origin of the normal-form equations (41-45) in finite time follows as a consequence of the construction procedure. Since stabilization of the normal-form equations to the origin is equivalent to stabilization of the state equations (25-29) to the origin, we conclude that the control inputs $u_{1}$ and $u_{2}$ given by Eq. (40) with $v_{1}$ and $v_{2}$ defined by the above control algorithm stabilize the reduced spacecraft dynamics described by Eqs. (25-29) to the equilibrium $\left(\omega_{1}, \omega_{2}, \phi, \theta, \psi\right)=(0,0,0,0,0)$ in finite time. A computer implementation of the feedback control strategy can be easily carried out.

\section{B. Feedback Stabilization Based on Rigid-Body}

\section{Rotational Characteristics}

We now present an alternate discontinuous feedback control strategy for stabilizing the origin of Eqs. (25-29) in finite time. This strategy requires that the spacecraft undergo a sequence of specified maneuvers and is based on the fact that rigid-body rotations do not commute. The physical interpretation of the sequence of maneuvers that transfers any initial state of Eqs. (25-29) to the origin is as follows:

1) Transfer the initial state of Eqs. (25-29) to any equilibrium state in finite time; i.e., bring the spacecraft to rest.

2) Transfer the resulting state to an equilibrium state where $\phi=0$ in finite time, i.e., so that the spacecraft is at rest with $\phi=0$.

3) Transfer the resulting state to an equilibrium state where $\phi=$ $0, \theta=0$ in finite time, i.e., so that the spacecraft is at rest with $\phi=0, \theta=0$.

4) Transfer the resulting state to an equilibrium state where $\phi=$ $\frac{1}{2} \pi, \theta=0$ in finite time, i.e., so that the spacecraft is at rest with $\phi=\frac{1}{2} \pi, \theta=0$.

5) Transfer the resulting state to the equilibrium state $\left(0,0, \frac{1}{2} \pi, 0,0\right)$ in finite time.

6) Transfer the equilibrium state $\left(0,0, \frac{1}{2} \pi, 0,0\right)$ to the equilibrium state $(0,0,0,0,0)$ in finite time.

We now present a feedback control algorithm that stabilizes the spacecraft to the origin in finite time; this feedback control algorithm implements the approach just described.

Maneuver 1. Apply

$$
\begin{aligned}
& u_{1}=-k \operatorname{sign} \omega_{1} \\
& u_{2}=-k \operatorname{sign} \omega_{2}
\end{aligned}
$$

until $\left(\omega_{1}, \omega_{2}\right)=(0,0)$; then go to maneuver 2 .

Maneuver 2. Apply

$$
\begin{gathered}
u_{1}=-G\left(\phi, \omega_{1}\right) \\
u_{2}=0
\end{gathered}
$$

until $\left(\omega_{1}, \omega_{2}, \phi\right)=(0,0,0)$; then go to maneuver 3 .
Maneuver 3. Apply

$$
\begin{gathered}
u_{1}=0 \\
u_{2}=-G\left(\theta, \omega_{2}\right)
\end{gathered}
$$

until $\left(\omega_{1}, \omega_{2}, \phi, \theta\right)=(0,0,0,0)$; then go to maneuver 4 . Maneuver 4. Apply

$$
\begin{gathered}
u_{1}=-G\left(\phi-\frac{1}{2} \pi, \omega_{1}\right) \\
u_{2}=0
\end{gathered}
$$

until $\left(\omega_{1}, \omega_{2}, \phi, \theta\right)=\left(0,0, \frac{1}{2} \pi, 0\right)$; then go to maneuver 5 . Maneuver 5. Apply

$$
\begin{gathered}
u_{1}=0 \\
u_{2}=-G\left(\psi, \omega_{2}\right)
\end{gathered}
$$

until $\left(\omega_{1}, \omega_{2}, \phi, \theta, \psi\right)=\left(0,0, \frac{1}{2} \pi, 0,0\right)$; then go to maneuver 6 . Maneuver 6. Apply

$$
\begin{gathered}
u_{1}=-G\left(\phi, \omega_{1}\right) \\
u_{2}=0
\end{gathered}
$$

until $\left(\omega_{1}, \omega_{2}, \phi, \theta, \psi\right)=(0,0,0,0,0,0)$; then go to maneuver 1 . It can be verified that the execution of maneuver 1 transfers the initial state of Eqs. (25-29) to the equilibrium state $\left(0,0, \phi^{1}, \theta^{1}, \psi^{1}\right)$ for some $\phi^{1}, \theta^{1}, \psi^{1}$ in finite time. Execution of maneuver 2 then transfers the state $\left(0,0, \phi^{1}, \theta^{1}, \psi^{1}\right)$ to the state $\left(0,0,0, \theta^{1}, \psi^{1}\right)$; execution of maneuver 3 then transfers the state $\left(0,0,0, \theta^{1}, \psi^{1}\right)$ to the state $\left(0,0,0,0, \psi^{1}\right)$; execution of maneuver 4 then transfers the state $\left(0,0,0,0, \psi^{1}\right)$ to the state $\left(0,0, \frac{1}{2} \pi, 0, \psi^{1}\right)$; execution of maneuver 5 then transfers the state $\left(0,0, \frac{1}{2} \pi, 0, \psi^{1}\right)$ to the state $\left(0,0, \frac{1}{2} \pi, 0,0\right)$; finally, execution of maneuver 6 transfers the state $\left(0,0, \frac{1}{2} \pi, 0,0\right)$ to the state $(0,0,0,0,0)$. This strategy is discontinuous and nonclassical in nature. A computer implementation of the feedback control strategy can be easily carried out.

\section{Comments}

We have introduced two different control laws that transfer any initial state of Eqs. (25-29) to the origin in finite time. Both control strategies are nonclassical and have been developed based on a careful study of the dynamics of the spacecraft system. Each of these control laws is in feedback form, since the control values depend on the current state; and each control law is discontinuous. The first construction procedure makes use of the nonholonomic features of the reduced spacecraft dynamics, whereas the second construction procedure uses physical insight about rigid-body rotations. The first control law constructed makes use of both control actuators simultaneously, whereas the second control law (after maneuver 1) uses only a single actuator at a time. The two discontinuous feedback control laws exhibited are illustrations of the class of control laws that asymptotically stabilize Eqs. (25-29) to the origin. There are other maneuver sequences, and corresponding feedback control laws, that will also achieve the desired attitude stabilization of the spacecraft. But each such strategy is necessarily discontinuous.

One of the advantages of the development in Secs. IV.A and IV.B is that feedback control strategies are constructed that guarantee attitude stabilization in a finite time. The total time required to complete the spacecraft reorientation is the sum of the times required to complete the sequence of maneuvers described. It should be clear that the time required to complete each maneuver depends on the single positive parameter $k$ in the corresponding control law. There is a trade-off between the required control levels, determined by the selection of $k$, and the resulting times to complete each of the maneuvers and hence the total time required to reorient the spacecraft. In particular, the time to reorient the spacecraft from a given initial state to the origin can be expressed as a function of the value of the parameter $k$ and of the initial state. 
We have demonstrated, by construction, the closed-loop properties for the special feedback control strategies presented. Our analysis was based on an ideal model assumption. Further robustness analysis is required to determine effects of model uncertainities and external disturbances. Unfortunately, such robustness analysis is quite difficult since the closed-loop vector fields are necessarily discontinuous. Perhaps, feedback control strategies that stabilize the spacecraft attitude, different from ones presented in this paper, would provide improved closed-loop robustness. These issues are to be studied in future research.

The Euler angle system used in this paper has a singularity when $\theta= \pm \frac{1}{2} \pi$. Therefore the results of the feedback control strategies are valid only in the region $-\pi \leq \psi \leq \pi,-\frac{1}{2} \pi<\theta<\frac{1}{2} \pi,-\pi$ $\leq \phi \leq \pi$. For the stabilization problem being considered in this paper, it is not clear how we could develop a control strategy that may be global (i.e., free of the Euler angle singularity) by introducing other representations such as quaternions for representing the orientation of the spacecraft. This is a subject of future research. To the best of our knowledge, there has been no previous work where an explicit control law is presented for reorientation of a spacecraft with just two momentum wheel actuators. Thus there are no previous results for comparison on the problem considered in this paper.

\section{Simulation}

We illustrate the results of the paper using an example. Consider a rigid spacecraft with no control torque about the third principal axis and two control torques, generated by momentum wheel actuators, are applied about the other two principal axes. Therefore the vectors $\boldsymbol{b}_{1}$ and $\boldsymbol{b}_{2}$ are given by $\boldsymbol{b}_{1}=(1,0,0)^{T}, \boldsymbol{b}_{2}=(0,1,0)^{T}$. For our simulation, we use the spacecraft parameters used in Ref. 2. The mass of the spacecraft, $m_{0}$, is $500 \mathrm{~kg}$, and the masses of the momentum wheels, $m_{1}$ and $m_{2}$, are each $5 \mathrm{~kg}$. The center of mass of the momentim wheels are located at a distance $0.2 \mathrm{~m}$ from the center of mass of the spacecraft, i.e., $d_{1}=d_{2}=0.2 \mathrm{~m}$. The moment of inertia of the wheels about its axis of rotation is $0.5 \mathrm{~kg} \cdot \mathrm{m}^{2}$, i.e., $j_{1}=j_{2}=0.5$. The inertial tensor of the spacecraft and the two momentum wheels are given as

$$
\begin{gathered}
\boldsymbol{I}_{0}=\operatorname{diag}(86.215,85.07,113.565) \mathrm{Kg} \cdot \mathrm{m}^{2} \\
\boldsymbol{I}_{1}=\operatorname{diag}(0.5,0.25,0.25) \mathrm{Kg} \cdot \mathrm{m}^{2} \\
\boldsymbol{I}_{2}=\operatorname{diag}(0.25,0.5,0.25) \mathrm{Kg} \cdot \mathrm{m}^{2}
\end{gathered}
$$

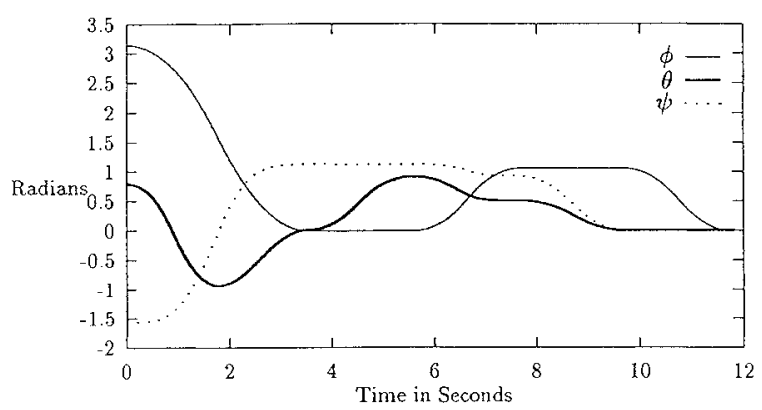

Fig. 2 Euler angles.

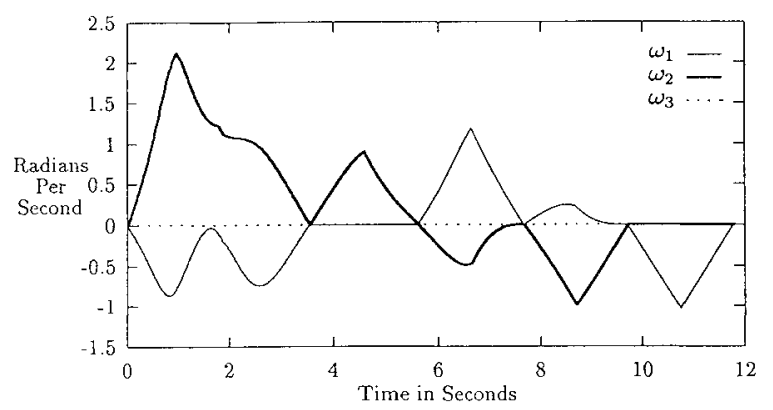

Fig. 3 Angular velocities.

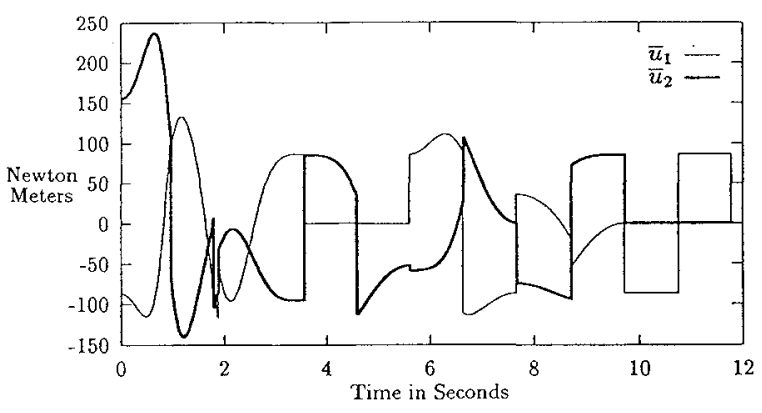

Fig. 4 Control torques.

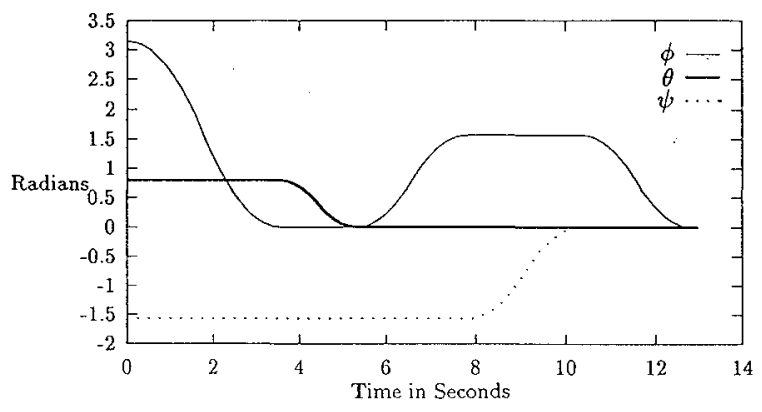

Fig. 5 Euler angles.

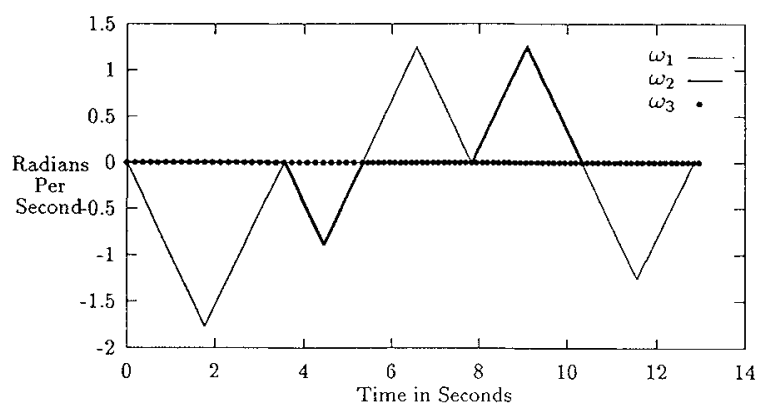

Fig. 6 Angular velocities.

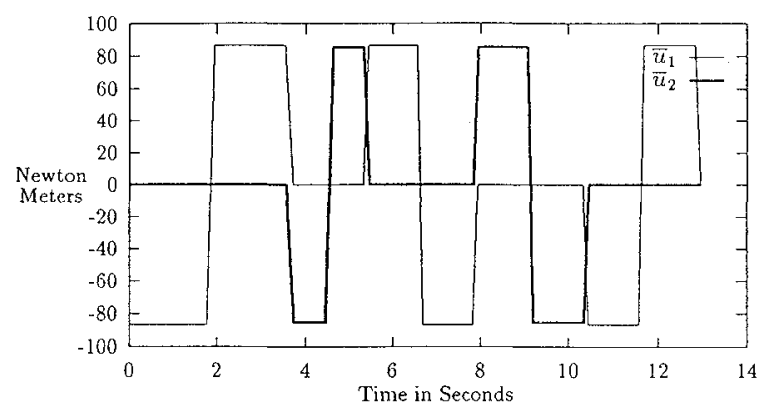

Fig. 7 Control torques.

Using these parameters, the inertial matrix $J$ can be calculated as

$$
J=\operatorname{diag}(86.7,85.5,114.5) \mathrm{Kg} \cdot \mathrm{m}^{2}
$$

approximately. The complete dynamics of the spacecraft system defined by Eqs. (19-22) is not controllable, but we consider the restriction that the angular momentum vector $\boldsymbol{H}=0$. Consequently, we are interested in stabilizing the reduced spacecraft dynamics described by Eqs. (25-29) to the equilibrium $\left(\omega_{1}, \omega_{2}, \phi, \theta, \psi\right)=$ $(0,0,0,0,0)$. The spacecraft is initially at rest (i.e., $\left.\omega^{0}=\omega_{2}^{0}=0\right)$ with an initial orientation given by the Euler angles $\phi^{0}=\pi, \theta^{0}=$ $0.25 \pi$, and $\psi^{0}=-0.5 \pi$.

First, a computer implementation of the feedback control algorithm specified in Sec. IV.A was used to stabilize the spacecraft to the origin. The value of the gain $k$ was chosen as 1 . The time responses of the Euler angles, angular velocities, and control torques are shown in Figs. 2, 3, and 4, respectively. After a total maneuver 
time of $11.77 \mathrm{~s}, \omega_{1}=\omega_{2}=\phi=\theta=\psi=0$. Next, a computer implementation of the feedback control algorithm specified in Section IV.B was used to stabilize the spacecraft to the origin. The value of the gain $k$ was chosen as 1 . The time responses of the Euler angles, angular velocities, and control torques are shown in Figs. 5, 6, and 7, respectively. After a total maneuver time of $13 \mathrm{~s}$, $\omega_{1}=\omega_{2}=\phi=\theta=\psi=0$.

\section{Conclusion}

The attitude stabilization problem of a spacecraft using control torques supplied by two momentum wheel actuators about axes spanning a two-dimensional plane orthogonal to a principal axis has been considered. The complete spacecraft dynamics are not controllable. However, the spacecraft dynamics are controllable under the restriction that the total angular momentum vector of the system is zero. The spacecraft dynamics under such a restriction cannot be asymptotically stabilized using time-invariant continuous feedback, but discontinuous feedback control strategies have been constructed that stabilize the spacecraft to any equilibrium attitude in finite time. The results of the paper show that it is possible to construct control laws based on a study of the particular spacecraft dynamics for reorienting a spacecraft using two momentum wheel actuators.

\section{Appendix}

From Eqs. (35-39), it is clear that $\omega_{1}=\omega_{2}=\phi=\theta=\psi=0$ implies that $y_{1}=y_{2}=y_{3}=y_{4}=y_{5}=0$. Consider the inversion of Eqs. (35-39). From Eq. (37), we have

$$
\phi=y_{3}
$$

From Eqs. (35) and (39) we have

$$
\left[\begin{array}{l}
y_{1} \\
y_{2}
\end{array}\right]=\left[\begin{array}{cc}
\cos \phi & \sin \phi \\
\sin \phi & -\cos \phi
\end{array}\right]\left[\begin{array}{c}
(\ln \sec \theta+\tan \theta) \\
\psi
\end{array}\right]
$$

Inverting the above equation, we get

$$
\psi=y_{1} \sin y_{3}-y_{5} \cos y_{3}
$$

and

$$
\ln (\sec \theta+\tan \theta)=y_{1} \cos y_{3}+y_{5} \sin y_{3}
$$

Therefore

$$
\sec \theta+\tan \theta=\exp \left(y_{1} \cos y_{3}+y_{5} \sin y_{3}\right)
$$

Now

$$
\sec \theta-\tan \theta=\frac{1}{\sec \theta+\tan \theta}
$$

Therefore

$$
\sec \theta-\tan \theta=\exp \left(-y_{1} \cos y_{3}-y_{5} \sin y_{3}\right)
$$

Thus we have

$$
\begin{gathered}
\theta=\tan ^{-1}\left\{0 . 5 \left[\exp \left(y_{1} \cos y_{3}+y_{5} \sin y_{3}\right)\right.\right. \\
\left.\left.-\exp \left(-y_{1} \cos y_{3}-y_{5} \sin y_{3}\right)\right]\right\}
\end{gathered}
$$

From Eqs. (36) and (38) we have

$$
\begin{gathered}
\omega_{2}=\frac{y_{2}+y_{4} y_{5}}{\sec \theta} \\
\omega_{1}=y_{4}-\frac{y_{2}+y_{4} y_{5}}{\sec \theta} \sin y_{3} \tan \theta
\end{gathered}
$$

where

$\tan \theta=0.5\left[\exp \left(y_{1} \cos y_{3}+y_{5} \sin y_{3}\right)-\exp \left(-y_{1} \cos y_{3}-y_{5} \sin y_{3}\right)\right]$

and

$\sec \theta=0.5\left[\exp \left(y_{1} \cos y_{3}+y_{5} \sin y_{3}\right)+\exp \left(-y_{1} \cos y_{3}-y_{5} \sin y_{3}\right)\right]$
Now it is clear that $y_{1}=y_{2}=y_{3}=y_{4}=y_{5}=0$ implies that $\omega_{1}=$ $\omega_{2}=\phi=\theta=\psi=0$. Moreover, the diffeomorphism is global in the interval $-\pi \leq \phi \leq \pi,-\frac{1}{2} \pi<\theta<\frac{1}{2} \pi,-\pi \leq \psi \leq \pi, \omega_{1} \in R$ and $\omega_{2} \in R$.

\section{Acknowledgments}

This work was partially supported by National Science Foundation Grant MSS-9114630 and by NASA Grant NAG-1-1419.

\section{References}

${ }^{1}$ Vadali, S. R., "Variable Structure Control of Spacecraft Large-Angle Maneuvers," Journal of Guidance, Control, and Dynamics, Vol. 9, No. 2, 1986, pp. 235-239.

${ }^{2}$ Vadali, S. R., and Junkins, J. L., "Optimal Open-Loop and Stable Feedback Control of Rigid Spacecraft Attitude Maneuvers," Journal of the Astronautical Sciences, Vol. 32, No. 2, 1984, pp. 105-122.

${ }^{3}$ Wen, J. T., and Kreutz-Delgado, K., "The Attitude Control Problem," IEEE Transactions on Automatic Control, Vol. 36, No. 10, 1991, pp. 11481161.

${ }^{4}$ Wie, B., and Barba, P. M., "Quaternion Feedback for Spacecraft Large Angle Maneuvers," Journal of Guidance, Control, and Dynamics, Vol. 8, No. 3, 1985, pp. 360-365.

${ }^{5}$ Meyer, G., "Design and Global Analysis of Spacecraft Attitude Control Systems," NASA Tech. Rept. R-361, March 1971.

${ }^{6}$ Dwyer, T. W. A., III, "Exact Nonlinear Control of Large Angle Rotational Maneuvers," IEEE Transactions on Automatic Control, Vol. 29, No. 9, 1984, pp. 769-774.

${ }^{7}$ Dwyer, T. W. A., III, "Exact Nonlinear Control of Spacecraft Slewing Maneuvers with Internal Momentum Transfers," Journal of Guidance, Control, and Dynamics, Vol. 9, No. 2, 1986, pp. 240-247.

${ }^{8}$ Crouch, P. E., "Spacecraft Attitude Control and Stabilization: Applications of Geometric Control Theory to Rigid Body Models," IEEE Transactions on Automatic Control, Vol. 29, No. 4, 1984, pp. 321-331.

${ }^{9}$ Byrnes, C. I., and Isidori, A., "On the Attitude Stabilization of Rigid Spacecraft," Automatica, Vol. 27, No. 1, 1991, pp. 87-95.

${ }^{10}$ Krishnan, H., McClamroch, N. H., and Reyhanoglu, M., "On the Attitude Stabilization of a Rigid Spacecraft Using Two Control Torques," Proceedings of the American Control Conference (Chicago, IL), 1992, pp. 19901995.

${ }^{11}$ Krishnan, H., Reyhanoglu, M., and McClamroch, N. H., "Attitude Stabilization of a Rigid Spacecraft Using Gas Jet Actuators Operating in a Failure Mode," Proceedings of the IEEE Conference on Decision and Control (Tucson, AZ), Inst. of Electrical and Electronics Engineers, 1992, pp. 1612-1617.

${ }^{12}$ Krishnan, H., Reyhanoglu, M., and McClamroch, N. H., "Attitude Stabilization of a Rigid Spacecraft Using Two Control Torques: A Nonlinear Control Approach Based on the Spacecraft Attitude Dynamics," Automatica, Vol. 30, No. 6, 1994, pp. 1023-1027.

${ }^{13}$ Tsiotras, P., and Longuski, J. M., "On Attitude Stabilization of Symmetric Spacecraft with Two Control Torques," Proceedings of the American Control Conference (San Francisco, CA), 1993, pp. 46-50.

${ }^{14}$ Aeyels, D., "Stabilization of a Class of Nonlinear Systems by a Smooth Feedback Control," Systems and Control Letters, No. 5, 1984, pp. 289-294.

${ }^{15}$ Aeyels, D., "Stabilization by Smooth Feedback of the Angular Velocity of a Rigid Body," Systems and Control Letters, No. 5, 1985, pp. 59-63.

${ }^{16}$ Aeyels, D., and Szafranski, M., "Comments on the Stabilizability of the Angular Velocity of a Rigid Body," Systems and Control Letters, No. 10, 1988, pp. 35-39.

${ }^{17}$ Brockett, R. W., "Asymptotic Stability and Feedback Stabilization," Differential Geometric Control Theory, edited by R. W. Brockett, R. S. Millman, and H. J. Sussman, Birkhauser, Boston, 1983, pp. 181-191.

${ }^{18}$ Sontag, E. D., and Sussman, H. J., "Further Comments on the Stabilizability of the Angular Velocity of a Rigid Body," Systems and Control Letters, No. 12, 1988, pp. 213-217.

${ }^{19}$ Bloch, A. M., Krishnaprasad, P. S., Marsden, J. E., and Sanchez de Alvarez, "Stabilization of Rigid Body Dynamics by Internal and External Torques," Automatica, Vol. 28, No. 4, 1992, pp. 745-756.

${ }^{20}$ Bloch, A. M., and Marsden, J. E., "Stabilization of Rigid Body Dynamics by the Energy-Casimir Method," Systems and Control Letters, No. 14, 1990 , pp. 341-346.

${ }^{21}$ Zhao, R., and Posbergh, T. A., "Stabilization of a Rotating Rigid Body by the Energy-Momentum Method," Proceedings of the IEEE Conference on Decision and Control (Tucson, AZ), Inst. of Electrical and Electronics Engineers, 1992, pp. 1583-1588.

${ }^{22}$ Wan, C. J., and Bernstein, D. S., "A Family of Optimal Nonlinear Feedback Controllers that Globally Stabilize Angular Velocity," Proceedings of the IEEE Conference on Decision and Control (Tucson, AZ), Inst. of Electrical and Electronics Engineers, 1992, pp. 1143-1148. 
${ }^{23}$ Outbib, R., and Sallet, G., "Stabilizability of the Angular Velocity of a Rigid Body Revisited," Systems and Control Letters, Vol. 18, 1992, pp. 93-98.

${ }^{24}$ Marsden, J. E., et. al., "Symmetry, Stability, Geometric Phases, and Mechanical Integrators (part II)," Nonlinear Science Today, Vol. 1.2, 1991, pp. 14-21.

${ }^{25}$ Krishnaprasad, P. S., "Lie-Poisson Structures, Dual-Spin Spacecraft and Asymptotic Stability," Nonlinear Analysis: Theory, Methods and Applications, Vol. 7, 1984, pp. 1011-1035.

${ }^{26}$ Wang, L. S., and Krishnaprasad, P. S., "Gyroscopic Control and Stabilization," Journal of Nonlinear Science, Vol. 2, 1992, pp. 367-415.

${ }^{27}$ Bloch, A. M., Reyhanoglu, M., and McClamroch, N. H., "Control and Stabilization of Nonholonomic Dynamic Systems," IEEE Transactions on Automatic Control, Vol. 37, No. 11, 1992, pp. 1746-1757.

${ }^{28}$ Greenwood, D., Principles of Dynamics, 2nd ed., Prentice-Hall, Englewood Cliffs, NJ, 1988.

${ }^{29}$ Nijmeijer, H., and Van der Schaft, A. J., Nonlinear Dynamical Control Systems, Springer-Verlag, New York, 1990.
${ }^{30}$ Sussman, H. J., "A General Theorem on Local Controllability," SIAM Journal on Control and Optimization, Vol. 25, 1987, pp. 158194.

${ }^{31}$ Sussman, H. J., "Local Controllability and Motion Planning for Some Classes of Systems with Drift," Proceedings of the IEEE Conference on Decision and Control (Brighton, England, UK), Inst. of Electrical and Electronics Engineers, 1991, pp. 1100-1114.

${ }^{32}$ Sontag, E. D., "Feedback stabilization of nonlinear systems," Robust Control of Linear Systems and Nonlinear Control, edited by M. A. Kaasshoek, J. H. Van Schuppen, and A. C. M. Ran. Birkhauser, Boston, 1990, pp. 61-81.

${ }^{33}$ Sussman, H. J., "Subanalytic Sets and Feedback Control," Journal of Differential Equations, Vol. 31, 1979, pp. 31-52.

${ }^{34}$ Pomet, J. B., "Explicit Design of Time-Varying Stabilizing Control Laws for a Class of Controllable Systems Without Drift," Systems and Control Letters, Vol. 18, 1992, pp. 147-158.

${ }^{35}$ Athans, M., and Falb, P. L., Optimal Control: An Introduction to the Theory and Its Applications, McGraw-Hill, New York, 1966. 